\title{
Welling-up of cerebrospinal fluid is a sign of remote supratentorial hemorrhage during microvascular decompression: a case report
}

Maidinamu Yakufujiang, Yoshinori Higuchi', Shogo Wakita, Kentaro Horiguchi, Shiro Ikegami and Yasuo Iwadate

\begin{abstract}
Background: Subdural hematoma (SDH) at a remote operative site is uncommon following neurosurgical procedures. However, supratentorial SDH is one of the rare complications following microvascular decompression (MVD). We report a case of supratentorial SDH following MVD. The welling of cerebrospinal fluid (CSF) in the cerebellomedullary fissure was observed unexpectedly during the dissection. It is a signal case that can improve our understanding of the occurrence of such rare complications and of possible mechanisms.

Case presentation: A 54-year-old woman was diagnosed with left hemifacial spasm (HFS) and had been receiving botulinum toxin injections since 10 years before surgery. CSF welling-up in the cerebellopontine cistern was noticed by the surgeon during the arachnoid dissection. MVD was performed completely. A thin supratentorial SDH was found on the right side (contralateral) on immediate postoperative computed tomography (CT). The SDH was asymptomatic, and the size of hematoma did not increase in follow-up CT scans after surgery. During the follow-up period, she was relieved of HFS. CSF welling-up can be considered the time of initiation of the hemorrhage.

Conclusion: Limited case reports of supratentorial SDH following MVD have been published, and none of them have reported time of initiation of the hemorrhage. The phenomenon of abnormal CSF welling-up in the cistern could be a sign of remote supratentorial hemorrhage.
\end{abstract}

Keywords: Cerebrospinal fluid, Lateral suboccipital approach, Microvascular decompression, Subdural hematoma, Complications

\section{Background}

Microvascular decompression (MVD) has become the treatment of choice for medication-resistant hemifacial spasm (HFS), trigeminal neuralgia, and glossopharyngeal neuralgia. The success rate is satisfactory; however, potential operative risks exist, especially postoperative hemorrhagic complications, including infratentorial and supratentorial hematomas, have been reported in the previous literatures [1-8]. Limited cases of supratentorial subdural hematoma (SDH) related to MVD have

* Correspondence: yhiguchi@faculty.chiba-u.jp

Department of Neurological Surgery, Chiba University Graduate School of Medicine, 1-8-1 Inohana, Chuo-ku, Chiba City, Chiba 260-8670, Japan been reported in the literature $[1,2,5,6,8-10]$. Most of the cases were asymptomatic; however, some cases involved severe sequelae. The mechanism of hemorrhage at the remote site remains controversial. The time of initiation of the remote event is often unknown, whether it initiated during the intradural procedure or after dural closure. If this time of initiation of the hemorrhage is known, coupled with a sign that a hemorrhage should be suspected, appropriate preventive measures and possible intervention can be planned. No detailed guides are available that can predict the time of initiation of hemorrhage so far. We report a case of supratentorial SDH following MVD, which presented as welling-up of cerebrospinal 
fluid (CSF) during the intracranial procedure, possibly indicating remote supratentorial hemorrhage.

\section{Case presentation}

A 54-year-old woman was diagnosed with left-side HFS and had been receiving botulinum toxin injections for 10 years before surgery. Preoperative magnetic resonance images (MRI) revealed the offending artery to be the posterior inferior cerebellar artery (PICA), which lies in close proximity to the area where the root of facial nerve exits the cranium (Fig. 1a). The patient underwent MVD via lateral suboccipital approach in the park-bench position, and no lumbar drainage was performed before surgery. During the dissection of cerebellomedullary cistern, abnormal amount of CSF welled up in the cistern. Accumulated CSF was removed using a suction tube for approximately $30 \mathrm{~s}$ (Fig. 2; Supplement video). Depth of general anesthesia was adequately maintained, and no coughing was observed. No hemorrhage was confirmed during surgery. PICA, the offending artery, was translocated to the petrosal bone and fixed with fibrin glue. The patient recovered from anesthesia normally without any neurological deficit. However, a small supratentorial SDH was found on the right side (contralateral) on immediate postoperative computed tomography (CT) scan (Fig. 1b). Since the SDH was asymptomatic, we decided to monitor the patient by follow-up CT scans. Any increase in the size of hematoma was not evident after surgery. During the follow-up period, she was relieved of HFS.

\section{Discussion}

We experienced a case of remote operative site supratentorial SDH following MVD. The patient was asymptomatic and was relieved of HFS during the follow-up period. Hemorrhagic complications following posterior fossa surgery have been described in several reports. These reports were mainly described in terms of surgical position, primary disease, and the site of hemorrhage. Surgery in a sitting position has been commonly associated with hemorrhagic complications after posterior fossa surgery.

There have been increasing cases of hemorrhagic complications after MVD in the recent years. Table 1 demonstrates previously reported cases of supratentorial SDH [1, 2, 6, 8-10]. Although most patients recovered without any deficits, unfavorable outcomes including mild facial numbness, gait disturbances [10], Gerstmann's syndrome, and epilepsy have also been reported [9]. The cause of this rare but serious complication should be known so that adequate preventive measures and accurate treatment protocols can be instituted.

Remote intracranial hemorrhage in the posterior fossa can also occur during supratentorial craniotomies. The reported incidence of remote cerebellar hemorrhage ranges from 0.04 to $0.8 \%$ [11]. The classic presentation of remote cerebellar hemorrhage on CT scans is intracerebellar and/or subarachnoid hemorrhage in the sulci of one or both cerebellar hemispheres and/or vermis on the superior aspect of the cerebellum [12]. The most common pattern of bleeding in remote cerebellar hemorrhage is subarachnoid bleeding along the cerebellar folia, which is rightly referred to as the "zebra sign" because of alternating dark and white patterns of blood and edema, respectively [13]. The underlying mechanism is still controversial; however, intraoperative CSF loss and stretching of the bridging veins are being considered as possible causes underlying this mechanism [11]. Preoperative antiplatelet agents and moderately elevated intraoperative systolic blood pressure are potentially
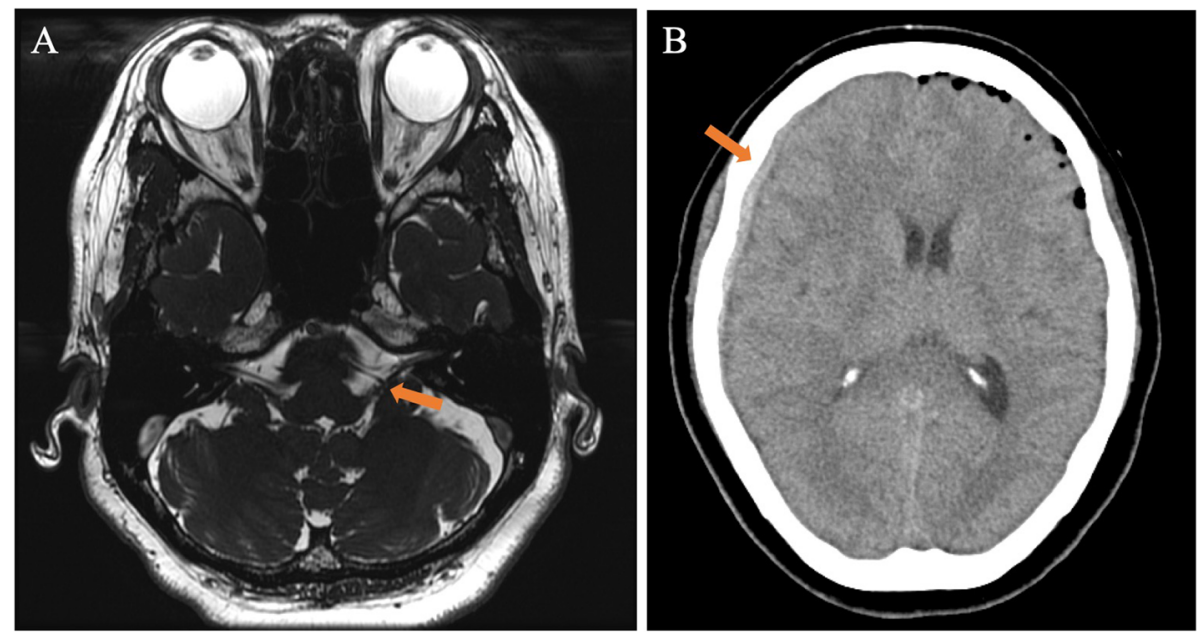

Fig. 1 a Preoperative magnetic resonance images. Arrow indicates the posterior inferior cerebellar artery adjacent to the zone of exit of the root of facial nerve. b Postoperative computed tomography scans showing unilateral supratentorial subdural hematomas (arrow) 


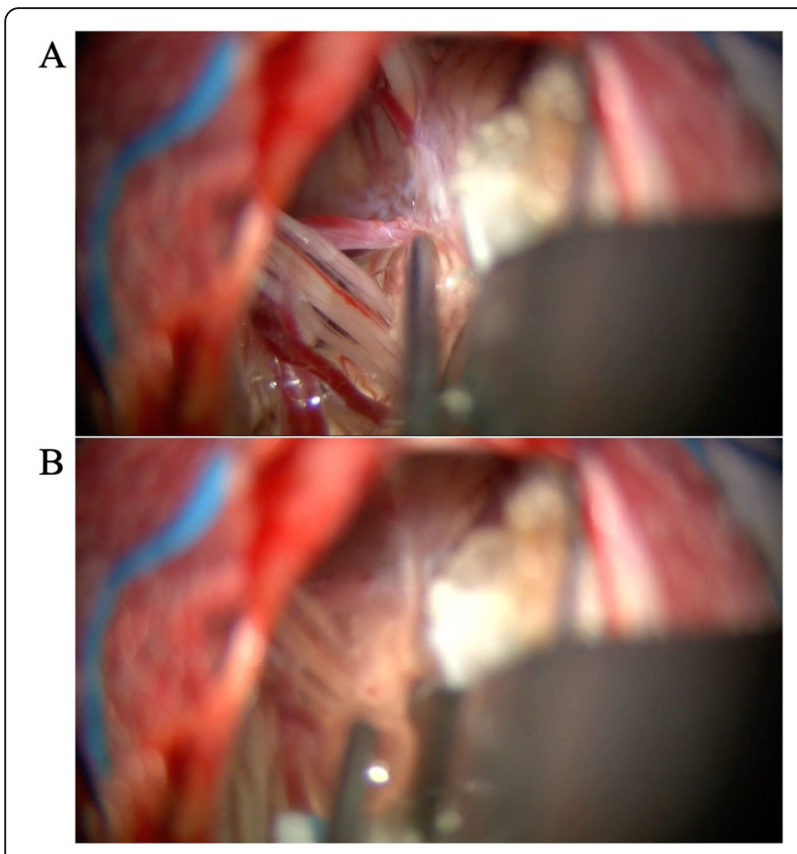

Fig. 2 Cerebrospinal fluid accumulation in the left cerebellopontine cistern during the surgery. $\mathbf{a}$ The photo before accumulation of cerebrospinal fluid. b After accumulation of cerebrospinal fluid. See the supplement video which is viewed stereoscopically

modifiable risk factors associated with the development of remote cerebellar hemorrhage [14].

For a relatively rare phenomenon, the mechanism of remote SDH following posterior fossa surgery is still controversial. Two principal possible mechanisms have been suggested. The first one suggests that excessive rapid removal of CSF from the posterior fossa could cause distortion of the supratentorial structures with laceration of the bridging veins, thereby inducing $\mathrm{SDH}$ [1]. The second mechanism was suggested by Li et al. in reports of three cases of delayed supratentorial SDH following MVD [5]. They suggested that the excessive rotation and flexion of the neck in the lateral decubitus position might obstruct jugular venous drainage on the contralateral side, resulting in venous hemorrhagic infraction in the supratentorial regions.

No detailed guides regarding the time of initiation of hemorrhage have been reported in previous studies. Nozaki et al. believed that hemorrhage could occur towards the end of the surgery. He hypothesized that during a change of position from lateral to supine, bilateral bridging veins being extended equally, the low intracranial pressure and/or intracranial air caused brain sagging and subsequently induced supratentorial SDH [8]. Intraoperative CSF welling-up has not been indicated as a sign of intraoperative supratentorial hemorrhage during the lateral suboccipital approach. The unexpected welling-up of the CSF might be induced by the small amount subdural hemorrhage. Subsequently, supratentorial ventricular CSF might flow out of the foramen of Luschka and Magendie. We advocate that this phenomenon might be related to remote supratentorial hemorrhage during the procedures. An immediate CT scan survey is recommended after surgery.

The current report has a limitation. A single case of abnormal rapid CSF accumulation in the cerebellomedullary cistern during surgery has been reported. Remote SDH was detected on postoperative CT scans in the previous reports. Intraoperative CT or MRI can provide direct evidence of the time of initiation of hemorrhage.

Table 1 Reports of supratentorial subdural hematoma following microvascular decompression

\begin{tabular}{|c|c|c|c|c|c|c|c|}
\hline Author/year & Age & Gender & Side & Primary disease & Side of hematoma & Required evacuation & Outcome \\
\hline Hanakita 1988 (ref [1]) & 67 & $\mathrm{~F}$ & $\mathrm{R}$ & HFS & Contralateral & No & No deficit \\
\hline Barker 1996 (ref [2]) & NA & NA & NA & TGN & NA & Yes & No deficit \\
\hline Oh 2008 (ref [6]) & NA & NA & NA & TGN & NA & No & No deficit \\
\hline \multirow[t]{2}{*}{ Li 2007 (ref [5]) } & 70 & M & L & HFS & Ipsilateral & Yes & Dead \\
\hline & 60 & $\mathrm{~F}$ & L & HFS & Ipsilateral & No & No deficit \\
\hline \multirow[t]{4}{*}{ Nozaki 2016 (ref [8]) } & 50 & $\mathrm{~F}$ & L & HFS & Contralateral & No & No deficit \\
\hline & 49 & $\mathrm{~F}$ & $\mathrm{R}$ & HFS & Ipsilateral & No & No deficit \\
\hline & 74 & $\mathrm{~F}$ & $\mathrm{R}$ & HFS & Ipsilateral & No & No deficit \\
\hline & 51 & M & L & HFS & Contralateral & No & No deficit \\
\hline \multirow[t]{3}{*}{ Amagasaki 2017 (ref [9]) } & 37 & M & $\mathrm{R}$ & HFS & Bilateral & Yes & Gerstmann's syndrome, epilepsy \\
\hline & 33 & M & L & HFS & Ipsilateral & No & No deficit \\
\hline & 25 & $\mathrm{~F}$ & L & HFS & Ipsilateral & No & No deficit \\
\hline \multirow[t]{2}{*}{ Kasuya 2018 (ref [10]) } & 55 & $\mathrm{~F}$ & NA & TGN & NA & No & Mild facial numbness \\
\hline & 63 & M & NA & TGN & NA & No & Gait disturbance \\
\hline
\end{tabular}




\section{Conclusion}

Most of the cases with remote supratentorial SDH achieved good outcomes; however, some of the cases have the possibility of encountering devastating results. To prevent this rare complication, previous studies have suggested that excessive CSF drainage should be avoided and that CSF loss should be compensated with sufficient amounts of artificial CSF immediately before dural closure. However, SDH at a remote operative site can occur even if the surgery was performed uneventfully. The phenomenon of abnormal CSF accumulation in the cerebellomedullary cistern should be considered a sign to suspect unexpected supratentorial hemorrhage, even if transient. An immediate postoperative CT scan is also recommended to detect the supratentorial hemorrhage in the patients who might need critical care and appropriate interventions.

\section{Supplementary information}

Supplementary information accompanies this paper at https://doi.org/10. 1186/s41984-020-00079-6.

Additional file 1: Supplement video. Cerebrospinal fluid welling-up in the left cerebellopontine cistern during the surgery. The video is viewed stereoscopically.

\section{Abbreviations}

CSF: Cerebrospinal fluid; CT: Computed tomography; HFS: Hemifacial spasm; MVD: Microvascular depression; PICA: Posterior inferior cerebellar artery; SDH: Subdural hematoma

\section{Acknowledgements}

None

\section{Authors' contributions}

MY and $\mathrm{YH}$ helped in the conception and design. MY and $\mathrm{YH}$ drafted the manuscript. SW, KH, SI, and IY critically revised the manuscript. All authors reviewed the final version of the manuscript and approved for submission.

\section{Funding}

No funding utilized from any organization.

\section{Availability of data and materials}

Data sharing not applicable to this article as no datasets were generated or analyzed during the current study.

\section{Ethics approval and consent to participate}

The ethical approval was not required from the institute as it is a case report. In our institute, for case report no need to take ethical approval. An informed consent was taken from the patient to participate in the report.

\section{Consent for publication}

The inform consent was taken from the patient for publication.

\section{Competing interests}

The authors declare that they have no competing interests.

Received: 5 October 2019 Accepted: 4 March 2020

Published online: 14 April 2020

\section{References}

1. Hanakita J, Kondo A. Serious complications of microvascular decompression operations for trigeminal neuralgia and hemifacial spasm. Neurosurgery. 1988;22:348-52.
2. Barker FG 2nd, Jannetta PJ, Bissonette DJ, Larkins MV, Jho HD. The longterm outcome of microvascular decompression for trigeminal neuralgia. $\mathrm{N}$ Engl J Med. 1996;334:1077-83.

3. McLaughlin MR, Jannetta PJ, Clyde BL, Subach BR, Comey CH, Resnick DK Microvascular decompression of cranial nerves: lessons learned after 4400 operations. J Neurosurg. 1999;90:1-8.

4. Kalkanis SN, Eskandar EN, Carter BS, Barker FG 2nd. Microvascular decompression surgery in the United States, 1996 to 2000: mortality rates, morbidity rates, and the effects of hospital and surgeon volumes. Neurosurgery. 2003:52:1251-61 discussion 1261-1252.

5. Li N, Zhao W, Pu C, Shen J. Delayed hemorrhage following microvascular decompression. Three case reports. Neurol Med Chir (Tokyo). 2007:47:186-8

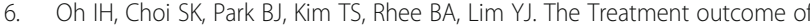
elderly patients with idiopathic trigeminal neuralgia: micro-vascular decompression versus gamma knife radiosurgery. J Korean Neurosurg Soc. 2008:44:199-204.

7. Dubey A, Sung WS, Shaya M, Patwardhan R, Willis B, Smith D, et al. Complications of posterior cranial fossa surgery--an institutional experience of 500 patients. Surg Neurol. 2009;72:369-75.

8. Nozaki T, Sugiyama K, Sameshima T, Kawaji H, Namba H. Supratentorial subdural hematoma following microvascular decompression: a report of four cases. Springerplus. 2016:5:353.

9. Amagasaki K, Takusagawa Y, Kanehashi K, Abe S, Watanabe S, Shono N, et al. Supratentorial acute subdural haematoma during microvascular decompression surgery: report of three cases. J Surg Case Rep. 2017; 2017:rjx004.

10. Kasuya H, Kuroi Y, Yokosako S, Koseki H, Tani S. Intraoperative and postoperative bleeding in microvascular decompression for trigeminal neuralgia. World Neurosurg. 2018;118:e123-8.

11. Park JS, Hwang JH, Park J, Hamm IS, Park YM. Remote cerebellar hemorrhage complicated after supratentorial surgery: retrospective study with review of articles. J Korean Neurosurg Soc. 2009;46:136-43.

12. Brockmann MA, Groden C. Remote cerebellar hemorrhage: a review. Cerebellum. 2006;5:64-8.

13. Brockmann MA, Nowak G, Reusche E, Russlies M, Petersen D. Zebra sign: cerebellar bleeding pattern characteristic of cerebrospinal fluid loss. Case report. J Neurosurg. 2005:102:1159-62.

14. Friedman JA, Piepgras DG, Duke DA, McClelland RL, Bechtle PS, Maher CO, et al. Remote cerebellar hemorrhage after supratentorial surgery. Neurosurgery. 2001;49:1327-40.

\section{Publisher's Note}

Springer Nature remains neutral with regard to jurisdictional claims in published maps and institutional affiliations.

\section{Submit your manuscript to a SpringerOpen ${ }^{\circ}$ journal and benefit from:}

- Convenient online submission

- Rigorous peer review

- Open access: articles freely available online

High visibility within the field

Retaining the copyright to your article

Submit your next manuscript at $\boldsymbol{\nabla}$ springeropen.com 